\title{
Robust 3D Segmentation of Pulmonary Nodules in Multislice CT Images
}

\author{
Kazunori Okada ${ }^{1}$, Dorin Comaniciu ${ }^{1}$, and Arun Krishnan ${ }^{2}$ \\ 1 Siemens Corporate Research, Inc. \\ 755 College Road East, Princeton, NJ 08540, USA \\ 2 Siemens Medical Solutions USA, Inc. \\ 51 Valley Stream Parkway, Malvern, PA 19355, USA
}

\begin{abstract}
We propose a robust and accurate algorithm for segmenting the 3D pulmonary nodules in multislice CT scans. The solution unifies i) the parametric Gaussian model fitting of the volumetric data evaluated in Gaussian scale-space and ii) non-parametric 3D segmentation based on normalized gradient (mean shift) ascent defining the basin of attraction of the target tumor in the $4 \mathrm{D}$ spatial-intensity joint space. This realizes the $3 \mathrm{D}$ segmentation according to both spatial and intensity proximities simultaneously. Experimental results show that the system reliably segments a variety of nodules including part- or non-solid nodules which poses difficulty for the existing solutions. The system also processes a $32 \times 32 \times 32$-voxel volume-of-interest efficiently by six seconds on average.
\end{abstract}

\section{Introduction}

One of the major goals of the computer-assisted diagnosis with the chest CT scans (chest CAD [1]) is reliable volumetric measurement of the pulmonary nodules [23]4]. Tumor change quantification based on such volume measurements plays an integral part of the cancer therapy monitoring and post-surgical examinations [5. There are a number of previous studies addressing the computerassisted volume measurement of nodules (e.g., Zhao et al. [6], Kostis et al. [5]). In these studies, the 2D or 3D tumor segmentation based on voxel intensity thresholding is used as the foundation of their solutions. Although such solutions are sufficient to delineate the well-defined solid nodules with the similar average intensity, they provide unreliable segmentations for the part- or non-solid nodules, as shown in Fig 1, A recent clinical study [7 has revealed that such nodules occur frequently and have a higher tendency to be malignant, motivating the development of the robust solution for these technically challenging cases.

This article proposes a novel 3D tumor segmentation method addressing the above issue. Our solution consists of two successive steps: i) 3D nodule center and spread estimation by fitting the anisotropic Gaussian intensity model in the Gaussian scale-space and ii) an iterative 3D nodule segmentation based on the basin of attraction in the $4 \mathrm{D}$ spatial-intensity joint space. The former step provides the reliable parametric estimation of the nodule's anisotropic structure by robustly fitting a Gaussian intensity model in the Gaussian scale-space of the 
given data 8,9. The latter step provides the non-parametric 3D nodule segmentation, according to both spatial and intensity proximities simultaneously, by using the normalized gradient ascent-based data segmentation in the $4 \mathrm{D}$ joint space. The results from the first step is interpreted as a normal prior and used to determine the analysis bandwidth of the latter step, resulting in an efficient segmentation solution. The joint-space segmentation that exploits the basin of attraction has provided a robust solution for the general image segmentation problem [10]. However, the method has not been considered in the medical imaging domain and provides an alternative segmentation principle to the intensity thresholding.

This article is organized as follows. Sec 2 and Sec 3 describe the first and the second step of our segmentation method, respectively. Sec 4 presents the results of our validation. This article is concluded by discussing our future work in Sec 5

\section{3D Tumor Center and Anisotropic Spread Estimation by Robust Scale-Space Analysis}

This section presents a robust estimation method for 3D tumor center location and anisotropic spread as the first reliable step towards the tumor segmentation. We assume that a marker $\mathbf{x}_{p}$, indicating the rough location of the target tumor, is given a priori. Such markers can be provided from an automatic tumor detection system (e.g., 11] ) or the screening results of radiologists and do not need to be accurate. Our solution is based on the anisotropic 3D Gaussian intensity model fitting in the Gaussian scale-space proposed in [8]. The following briefly describes this solution.

The volumetric CT data is formalized as a continuous positive function $I: \mathcal{R}_{+}^{3} \rightarrow \mathcal{R}_{+}$over the data space $\mathbf{x}=\left(x_{1}, x_{2}, x_{3}\right)$. A local region of $I(\mathbf{x})$ around a spatial extremum $\mathbf{u}$, expressing a pulmonary tumor, is modeled by the anisotropic 3D Gaussian intensity model,

$$
\begin{aligned}
I(\mathbf{x}) & \simeq \alpha \times\left.\Phi(\mathbf{x} ; \mathbf{u}, \mathbf{\Sigma})\right|_{\mathbf{x} \in \mathcal{S}} \\
\Phi(\mathbf{x} ; \mathbf{u}, \boldsymbol{\Sigma}) & =(2 \pi)^{-d / 2}|\boldsymbol{\Sigma}|^{-1 / 2} \exp \left(-\frac{1}{2}(\mathbf{x}-\mathbf{u})^{t} \boldsymbol{\Sigma}^{-1}(\mathbf{x}-\mathbf{u})\right)
\end{aligned}
$$

where $\alpha$ is an amplitude parameter, $\boldsymbol{\Sigma}$ is a fully-parameterized $3 \times 3$ symmetric positive definite covariance matrix, and $\mathcal{S}$ is a set of data points in the neighborhood of $\mathbf{u}$, belonging to the basin of attraction of $\mathbf{u}$. The mean $\mathbf{u}$ and covariance $\boldsymbol{\Sigma}$ of $\Phi$ describes the tumor location and spread, respectively. Thus the problem of our interest can be understood as parametric model fitting or robust estimation of $(\mathbf{u}, \boldsymbol{\Sigma})$ given $I(\mathbf{x})$. Anisotropy of the tumor can only be described by considering the estimation of the fully-parameterized covariance.

Multi-scale analysis is employed for this estimation, given a set of ordered and densely sampled analysis scales $\left\{h_{k} \mid k=1, . ., K\right\}$. The model mean and covariance are robustly estimated, by the method described below, for each analysis scale $h_{k}$, resulting in a set of successive estimates $\left\{\left(\mathbf{u}_{k}, \boldsymbol{\Sigma}_{k}\right)\right\}$. The final result 

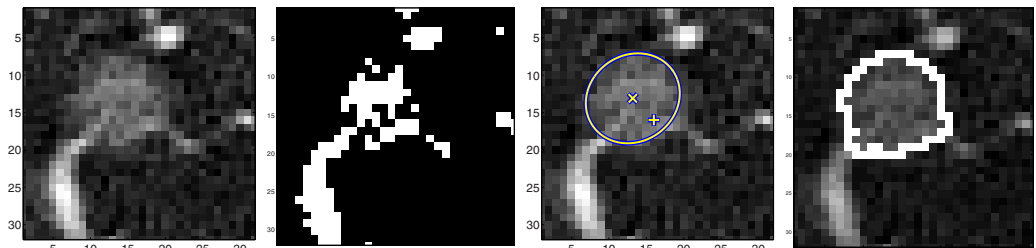

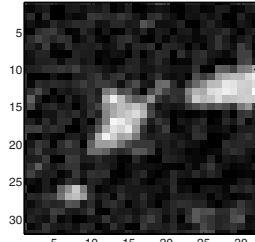

(a)

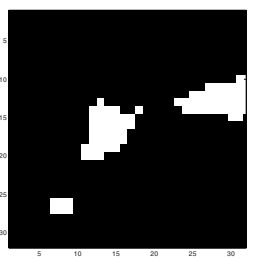

(b)

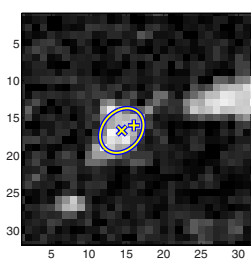

(c)

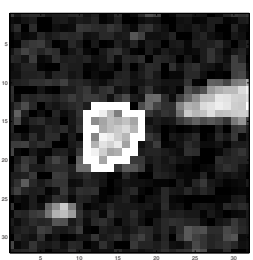

(d)

Fig. 1. 2D examples of the pulmonary nodule segmentation. From left to right, (a): 2D profile of two nodule examples, (b): segmentation results by the FWHM intensity thresholding, (c): center $(x)$ and anisotropic spread (ellipse) estimated by our method (+ indicates the marker location $\mathbf{x}_{p}$ described in Sec. 22), (d): nodule segmentation result by our method without any geometrical post-processings. The first row is an example of the part- and non-solid nodules while the second row is of the solid nodules. Both methods provides similar segmentation results for the solid case, however, the intensity thresholding method fails for the non-solid case. The maximum intensity for (b) was computed using the prior information shown in (c).

is given by finding the most stable estimate using a divergence-based stability test. The most stable estimate $\left(\mathbf{u}^{*}, \mathbf{\Sigma}^{*}\right)$ is defined as the estimate with the scale $h^{*}$ that assumes a local minimum of the modified Jensen-Shannon divergence profile over the scales [12,8] At each scale $h_{k}$, the divergence is computed over three neighboring scales.

For each analysis bandwidth $h_{k},\left(\mathbf{u}_{k}, \boldsymbol{\Sigma}_{k}\right)$ are estimated by scale-space mean shift analysis together with the robust estimation technique based on the basin of attraction. Gaussian scale-space of $I(\mathbf{x})$, or the solution of the diffusion equation $\partial_{h} I=\frac{1}{2} \nabla^{2} I$, is defined by a convolution of $I(\mathbf{x})$ with a set of Gaussian kernels with the analysis scales $\{h>0\}, L(\mathbf{x} ; \mathbf{H})=f(\mathbf{x}) * \Phi(\mathbf{x} ; \mathbf{0}, \mathbf{H})$, where $\mathbf{H}$ is an isotropic bandwidth matrix of a form $\mathbf{H}=h \mathbf{I}$. Its gradient vector is given by,

$$
\begin{aligned}
\nabla L(\mathbf{x} ; \mathbf{H}) & =I(\mathbf{x}) * \nabla \Phi(\mathbf{x} ; \mathbf{0}, \mathbf{H})=\mathbf{H}^{-1} L(\mathbf{x} ; \mathbf{H}) \mathbf{m}(\mathbf{x} ; \mathbf{H}) \\
\mathbf{m}(\mathbf{x} ; \mathbf{H}) & \equiv \frac{\int \mathbf{x}^{\prime} \Phi\left(\mathbf{x}-\mathbf{x}^{\prime} ; \mathbf{H}\right) f\left(\mathbf{x}^{\prime}\right) d \mathbf{x}^{\prime}}{\int \Phi\left(\mathbf{x}-\mathbf{x}^{\prime} ; \mathbf{H}\right) f\left(\mathbf{x}^{\prime}\right) d \mathbf{x}^{\prime}}-\mathbf{x}=\mathbf{H} \frac{\nabla L(\mathbf{x} ; \mathbf{H})}{L(\mathbf{x} ; \mathbf{H})}
\end{aligned}
$$

The vector $\mathbf{m}(\mathbf{x})$ is called scale-space mean shift vector and proportional to the gradient vector $\nabla L(\mathbf{x} ; \mathbf{H})$. A convergent iterative algorithm 12 for the normalized gradient ascent in the scale-space, $\mathbf{x}_{k+1}=\mathbf{m}\left(\mathbf{x}_{k}\right)+\mathbf{x}_{k}$, is used to estimate the tumor center $\mathbf{u}_{k}$, to which the given marker $\mathbf{x}_{p}$ converges. To increase the robustness, a set of the gradient ascents are performed from different initial points sampled uniformly around $\mathbf{x}_{p}$. The convergence point of the majority of the initial points defines the center estimate $\mathbf{u}_{k}$. 
Next, given the center estimate, the corresponding covariance $\boldsymbol{\Sigma}_{k}$ is estimated. Substituting the Gaussian tumor model (1) to the definition of the scalespace mean shift (4) reveals that the mean shift can be expressed as a quasi-linear matrix equation after some algebra, $\mathbf{m}(\mathbf{x} ; \mathbf{H})=\mathbf{H}(\boldsymbol{\Sigma}+\mathbf{H})^{-1}(\mathbf{u}-\mathbf{x})$. An overcomplete set of the linear equations with unknown $\boldsymbol{\Sigma}$ is constructed by using the mean shift vectors sampled within the basin of attraction of the target tumor. For this, we perform a set of the mean shift iterations from different initial points that are sampled uniformly around the center estimate $\mathbf{u}_{k} \cdot N_{u}$ mean shift vectors along convergent trajectories are used for constructing the overcomplete system,

$$
\begin{aligned}
\mathbf{A} \boldsymbol{\Sigma} & =\mathbf{B}, \boldsymbol{\Sigma} \in \mathcal{S P D} \\
\mathbf{A} & =\left(\mathbf{m}\left(\mathbf{x}_{1} ; \mathbf{H}\right), . ., \mathbf{m}\left(\mathbf{x}_{N_{u}} ; \mathbf{H}\right)\right)^{t} \mathbf{H}^{-t} \\
\mathbf{B} & =\left(\mathbf{b}_{1}, . ., \mathbf{b}_{N_{u}}\right)^{t}, \mathbf{b}_{j}=\mathbf{u}_{k}-\mathbf{x}_{j}-\mathbf{m}\left(\mathbf{x}_{j} ; \mathbf{H}\right)
\end{aligned}
$$

where $\mathcal{S P D}$ denotes a set of all symmetric positive definite matrices in $\mathcal{R}^{3 \times 3}$. A closed-form solution of this constrained system is given by minimizing an area criterion $\left\|\mathbf{A} \mathbf{Y}-\mathbf{B} \mathbf{Y}^{-t}\right\|_{F}^{2}$ where $\mathbf{Y}$ is Cholesky factorization of $\boldsymbol{\Sigma}=\mathbf{Y} \mathbf{Y}^{t}$ and $\|\cdot\|_{F}$ is the Frobenius matrix norm. The solution is expressed by a function of symmetric Schur decompositions of $\mathbf{P} \equiv \mathbf{A}^{t} \mathbf{A}$ and $\tilde{\mathbf{Q}} \equiv \boldsymbol{\Sigma}_{P} \mathbf{U}_{P}^{t} \mathbf{Q} \mathbf{U}_{P} \boldsymbol{\Sigma}_{P}$ given $\mathbf{Q} \equiv \mathbf{B}^{t} \mathbf{B}$

$$
\begin{aligned}
\boldsymbol{\Sigma}_{k} & =\mathbf{U}_{P} \boldsymbol{\Sigma}_{P}^{-1} \mathbf{U}_{\tilde{Q}} \boldsymbol{\Sigma}_{\tilde{Q}} \mathbf{U}_{\tilde{Q}}^{t} \boldsymbol{\Sigma}_{P}^{-1} \mathbf{U}_{P}^{t} \\
\mathbf{P} & =\mathbf{U}_{P} \boldsymbol{\Sigma}_{P}^{2} \mathbf{U}_{P}^{t}, \\
\tilde{\mathbf{Q}} & =\mathbf{U}_{\tilde{Q}} \boldsymbol{\Sigma}_{\tilde{Q}}^{2} \mathbf{U}_{\tilde{Q}}^{t}
\end{aligned}
$$

The robustness of the solution is endowed by using the information only within the basin of attraction, which effectively suppresses outliers.

This parametric estimation step yields the estimates of the $3 \mathrm{D}$ tumor center and tumor spread in the form of $3 \mathrm{D}$ mean vector $\mathbf{u}^{*}$ and $3 \times 3$ covariance matrix $\Sigma^{*}$ in (1). Also provided is the bandwidth $h^{*}$ that yields the above estimates which are most stable among others. The center and spread estimates can be interpreted as the normal probability distribution $g(\mathbf{x})$ of the center estimate,

$$
g(\mathbf{x})=\mathcal{N}\left(\mathbf{x} ; \mathbf{u}^{*}, \mathbf{\Sigma}^{*}\right)=\frac{1}{\left|2 \pi \boldsymbol{\Sigma}^{*}\right|^{1 / 2}} \exp \left(-\frac{1}{2}\left(\mathbf{x}-\mathbf{u}^{*}\right)^{t} \mathbf{\Sigma}^{*-1}\left(\mathbf{x}-\mathbf{u}^{*}\right)\right)
$$

\section{3D Tumor Segmentation Based on Basin of Attraction in $4 \mathrm{D}$ Spatial-Intensity Joint Space}

This section presents our solution for the non-parametric 3D nodule segmentation based on defining the basin of attraction of the target nodule in the 4D spatial-intensity joint space. The solution exploits the normal prior from the previous step, improving the efficiency of the original method proposed in $[10$.

The spatial-intensity joint space is conceived by interpreting the $3 \mathrm{D}$ function as a set of data points in a $4 \mathrm{D}$ space. This is achieved by introducing, to the $3 \mathrm{D}$ data space $\mathbf{x} \in \mathcal{R}_{+}^{3}$, another orthogonal dimension for the distribution of 
the function responses, resulting in the joint space $\mathbf{y} \equiv(\mathbf{x}, I(\mathbf{x})) \in \mathcal{R}_{+}^{4}$. A volumetric CT data is a discretization of the function $I(\mathbf{x})$ over a $3 \mathrm{D}$ regular lattice, resulting $N$ data locations $\left\{\mathbf{x}_{i} \in \mathcal{Z}_{+}^{3} \mid i=1, . ., N\right\}$ where $N=\prod_{d=1}^{3} N_{d}$ and $N_{d}$ is the number of voxels along the dimension $d$. Therefore, in the spatialintensity joint space, the discretized samples $\left\{I\left(\mathbf{x}_{i}\right)\right\}$ is interpreted as a set of $4 \mathrm{D}$ data points $\left\{\mathbf{y}_{i} \equiv\left(\mathbf{x}_{i}, I\left(\mathbf{x}_{i}\right)\right\}\right.$. The sample density estimate with normal kernel with a $4 \times 4$ bandwidth matrix $\mathbf{H}$ is given at a data point $\mathbf{y}$ by,

$$
f(\mathbf{y})=\frac{1}{N|2 \pi \mathbf{H}|^{1 / 2}} \sum_{i=1}^{N} \exp \left(-\frac{1}{2}\left(\mathbf{y}-\mathbf{y}_{i}\right)^{t} \mathbf{H}^{-1}\left(\mathbf{y}-\mathbf{y}_{i}\right)\right)
$$

Consequently, the gradient of the density $f(\mathbf{y})$ is given by,

$$
\begin{aligned}
\nabla f(\mathbf{y}) & =\frac{1}{N|2 \pi \mathbf{H}|^{1 / 2}} \sum_{i=1}^{N} \mathbf{H}^{-1}\left(\mathbf{y}_{i}-\mathbf{y}\right) \exp \left(-\frac{1}{2}\left(\mathbf{y}-\mathbf{y}_{i}\right)^{t} \mathbf{H}^{-1}\left(\mathbf{y}-\mathbf{y}_{i}\right)\right) \\
& =\mathbf{H}^{-1} f(\mathbf{y}) \mathbf{m}(\mathbf{y}) \\
\mathbf{m}(\mathbf{y}) & \equiv \frac{\sum_{i=1}^{N} \mathbf{y}_{i} \exp \left(-\frac{1}{2}\left(\mathbf{y}-\mathbf{y}_{i}\right)^{t} \mathbf{H}^{-1}\left(\mathbf{y}-\mathbf{y}_{i}\right)\right)}{\sum_{i=1}^{N} \exp \left(-\frac{1}{2}\left(\mathbf{y}-\mathbf{y}_{i}\right)^{t} \mathbf{H}^{-1}\left(\mathbf{y}-\mathbf{y}_{i}\right)\right)}-\mathbf{y}=\mathbf{H} \frac{\nabla f(\mathbf{y})}{f(\mathbf{y})}
\end{aligned}
$$

The vector $\mathbf{m}(\mathbf{y})$ is the density mean shift in the $4 \mathrm{D}$ joint space. A convergent iterative algorithm for the normalized density gradient ascent is obtained by,

$$
\mathbf{y}_{k+1}=\mathbf{m}\left(\mathbf{y}_{k}\right)+\mathbf{y}_{k}
$$

The iterator (16) is employed to cluster the data points according to both spatial and intensity proximities simultaneously. The points belonging to the basin of attraction of the target nodule are detected by applying (16) from a set of initial points, sampled according to the normal prior in (11), until convergence at $\nabla f(\mathbf{y})=0$. Initial points are sampled within a confidence interval of the 3D normal distribution between $p_{l o}$ and $p_{u p}$ percentiles. The points that converge to the vicinity of $\left(\mathbf{u}^{*}, m_{I}\right)$ are merged into a cluster which defines the target nodule. The points with the probability above $p_{u p}$ are also considered to be a part of the nodule. For each point $\mathbf{y}_{i}$ in the joint space, there is only one corresponding point $\mathbf{x}_{i}$ in the $3 \mathrm{D}$ data space. Thus, the cluster membership of $\mathbf{y}_{i}$ is directly associated with the data point $\mathbf{x}_{i}$, resulting in the segmentation of the tumor and background in the data space $\mathbf{x}$.

The advantages of this method include i) robust segmentation without intensity thresholding and ii) its insensitivity to variation of the intensity range, in comparison with the global threshold-based approach. However, for achieving the robustness in the segmentation results, it is crucial that the kernel bandwidth $\mathbf{H}$ is set appropriately for a given data $\left\{I\left(\mathbf{x}_{i}\right)\right\}$. Our solution determines $\mathbf{H}$ by exploiting the normal prior. $\mathbf{H}$ is formed as a diagonal matrix with the most stable bandwidth $h^{*}$ and the variance estimate of the intensities $\sigma_{I}^{2}$,

$$
\mathbf{H}=\operatorname{diag}\left(h^{*}, h^{*}, h^{*}, \sigma_{I}^{2}\right)
$$


where $\sigma_{I}^{2}$ is given by the sample variance of the intensity values within a $q$ percentile confidence ellipsoid of the normal distribution $g(\mathbf{x})$,

$$
\sigma_{I}^{2}=\left.\frac{1}{N_{\sigma}} \sum_{i=1}^{N_{\sigma}}\left(I\left(\mathbf{x}_{i}\right)-m_{I}\right)^{2}\right|_{\mathbf{x}_{i} \in\left\{\mathbf{x}_{i} \mid\left(\mathbf{x}_{i}-\mathbf{u}^{*}\right)^{t} \boldsymbol{\Sigma}^{*-1}\left(\mathbf{x}_{i}-\mathbf{u}^{*}\right)<c\right\}}
$$

The sample mean of the set of the intensity values and the number of voxels within the confidence ellipsoid are denoted by $m_{I}$ and $N_{\sigma}$, respectively. The parameter $c$ is directly derived from the specific choice of the percentile $q$. The segmentation procedure using (16) is carried out using the mean shift vectors computed with the resulting bandwidth matrix.

\section{Experimental Results}

The proposed segmentation method is evaluated with a database of clinical multislice chest CT scans with $1 \mathrm{~mm}^{2} \times 1.5 \mathrm{~mm}$ slice thickness, containing 77 nodules of 14 patients. The size of the nodules ranges between $3 \mathrm{~mm}$ to $25 \mathrm{~mm}$ in diameter. The data is also provided with the markers $\mathbf{x}_{p}$ and the classification labels for the part- or non-solid nodules given by radiologists. The database includes i) 6 cases of the part- or non-solid nodules, ii) 28 cases of small nodules whose size is less than $5 \mathrm{~mm}$, iii) 20 cases of nodules attached to the pleural surface, iv) 12 cases of largely non-spherical (anisotropic) nodules. An implementation of the above method is instantiated with the following settings. For the scale-space anisotropic intensity model fitting, a set of 25 analysis scales $h=\left\{0.5^{2}, 0.75^{2}, . ., 6.5^{2}\right\}$ are used. For the $4 \mathrm{D}$ joint space segmentation, the confidence limits for sampling the initial points and for estimating the sample intensity variance are set to $p_{l o}=25 \%, p_{u p}=75 \%$, and $q=75 \%$, respectively.

The performance evaluation of the system resulted in the correct parametric fits and non-parametric segmentations for 69 nodules by experts inspections. The 8 failures were due to i) very small nodules attached to pleural surface (6 cases), ii) very small vascularized nodule (1 case), iii) extremely elongated nodule (1 case). All the part- or non-solid and solitary small nodules were correctly estimated and segmented. The rejection criterion based on chi-square residual analysis [8] is applied, resulting successful rejection of all the failure cases attached to pleural surfaces. Fig. illustrates examples of the results. Each image is a $2 \mathrm{D}$ dissection of the target volume intersecting the estimated nodule center. The estimation results from the first step are visualized as an intersection of $50 \%$-confidence ellipsoid of the normal prior (11). More results can be found at http://www.scr.siemens.com/anisotropic/. The system's sensitivity to the initial marker locations is studies by randomly perturbing the markers within the $50 \%$-confidence limit range, using 36 nodules. The average error of the mean and covariance estimates from total average of the perturbation were 1.12 voxel and 8.21 Frobenius matrix norm, respectively. The results show the robustness of our method against the uncertainty of the marker locations. 
(1)
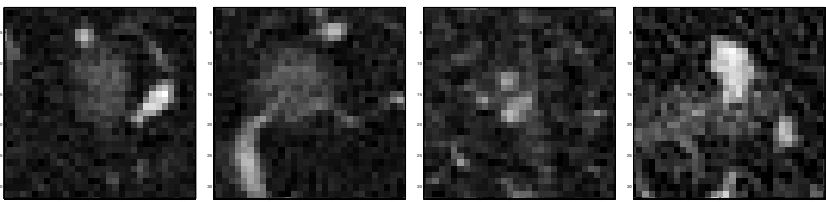

(2)
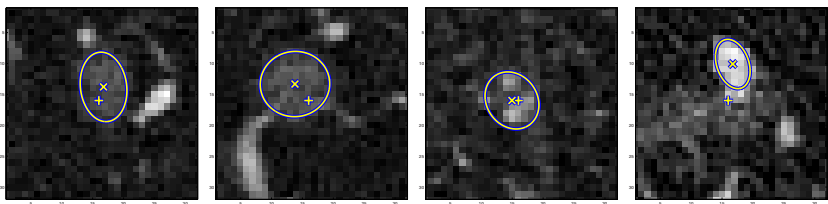

(3)
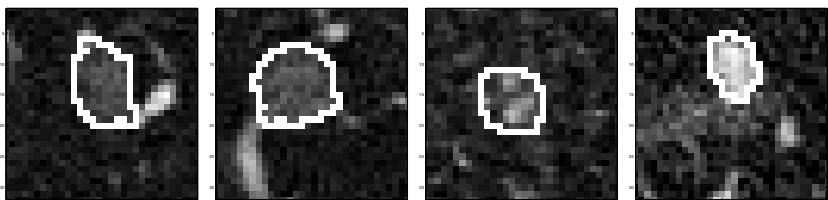

(a)

(1)

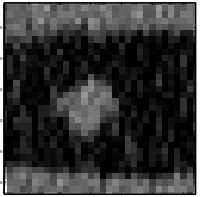

(2)

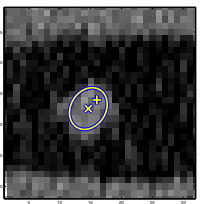

(3)

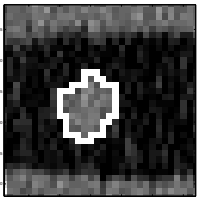

(e) (b)
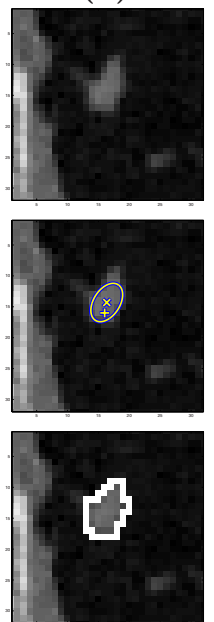

(f) (c)
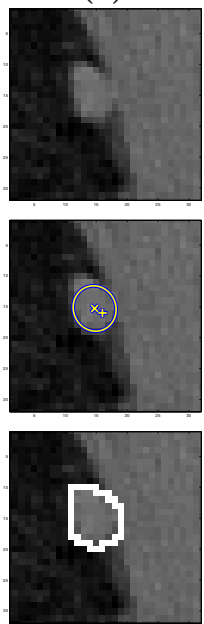

$(\mathrm{g})$ (d)
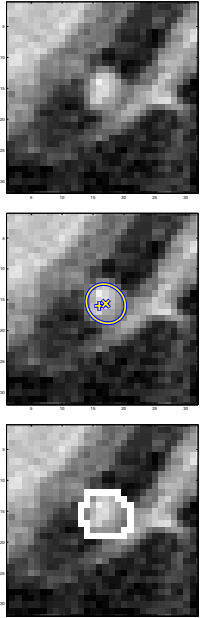

(h)

Fig. 2. Examples of the 3D estimation and segmentation results. The results are projected to a $2 \mathrm{D}$ plane for visualization. (1): $2 \mathrm{D}$ profile of input nodules, (2): parametric fitting results ( "+": $\mathbf{x}_{p}$, " $\times$ ": $\mathbf{u}^{*}$, ellipse: $\left.\boldsymbol{\Sigma}^{*}\right)$. (3): non-parametric segmentation results. (a)-(b): non-solid, (c): part-solid, (d)-(f): anisotropic, (g)-(h): pleural attachments. Our method flexibly refines the nodule shape approximated by a Gaussian in (2) to the non-parametric segmentation in (3) (see cases $(\mathrm{d}-\mathrm{g})$ ). The method provides reliable segmentation even in the presence of neighboring structures (see cases (b), (d), $(\mathrm{g}-\mathrm{h}))$. 


\section{Conclusions}

We proposed a robust and accurate method for segmenting the 3D pulmonary nodules in multislice CT scans. Our solution unifies the parametric and nonparametric algorithms, realizing accurate and efficient 3D segmentation according to both spatial and intensity proximities simultaneously. The parametric model fitting in the first step realizes robust characterization of tumor's anisotropic structures, while the non-parametric segmentation in the second step refines the results for finding more accurate 3D tumor boundary. The method provides reliable 3D segmentation of a variety of nodules including the clinically significant small and part- or non-solid nodules. The system implemented in $\mathrm{C}$ language segments the nodules efficiently. It processes a 32-voxel cubic volumeof-interest 6 seconds on average using an off-the-shelf PC with a $2.4 \mathrm{GHz}$ Intel CPU. Our future work includes i) further validation of the proposed method with more data and for volumetric measurements, ii) system optimization for enhancing the efficiency towards realizing real-time clinical applications, and iii) improvement of the segmentation results by accounting for the partial volume effect.

Acknowledgments. The authors wish to thank Visvanathan Ramesh from Siemens Corporate Research for stimulating discussions, Alok Gupta from CAD group, Siemens Medical Solutions, for his support and encouragement, and Jonathan Stoeckel from CAD group, Siemens Medical Solutions, for his valuable technical supports.

\section{References}

1. Reeves, A.P., Kostis, W.J.: Computer-aided diagnosis of small pulmonary nodules. Seminars in Ultrasound, CT, and MRI 21 (2000) 116-128

2. Ko, J.P., Rusinek, H., Jacobs, E.L., Babb, J.S., Betke, M., McGuinness, G., Naidich, D.P.: Small pulmonary nodules: Volume measurement at chest CT phantom study. Radiology 228 (2003) 864-870

3. Ko, J.P., Naidich, D.P.: Lung nodule detection and characterization with multislice CT. Radiol. Clin. N. Am. 41 (2003) 575-597

4. Wormanns, D., Kohl, G., Kotz, E., Marheine, A., Beyer, F., Heindel, W., Diederich, S.: Volumetric measurements of pulmonary nodules at multi-row detector CT : in vivo reproducibility. Eur. Radiol. 14 (2004) 86-92

5. Kostis, W.J., Reeves, A.P., Yankelevitz, D.F., Henschke, C.I.: Three-dimensional segmentation and growth-rate estimation of small pulmonary nodules in helical CT images. IEEE Trans. Medical Imaging 22 (2003) 1259-1274

6. Zhao, B., Yankelevitz, D., Reeves, A., Henschke, C.: Two-dimensional multicriterion segmentation of pulmonary nodules on helical CT images. IEEE Trans. Medical Imaging 22 (2003) 1259-1274

7. Henschke, C.I., Yankelevitz, D.F., Mirtcheva, R., McGuinness, G., McCauley, D., Miettinen, O.S.: CT screening for lung cancer: frequency and significance of partsolid and non-solid nodules. AJR Am. J. Roentgenol. 178 (2002) 1053-1057 
8. Okada, K., Comaniciu, D., Dalal, N., Krishnan, A.: A robust algorithm for characterizing anisotropic local structures. In: Euro. Conf. Computer Vision. (2004)

9. Okada, K., Comaniciu, D., Krishnan, A.: Scale selection for anisotropic scale-space: Application to volumetric tumor characterization. In: IEEE Conf. Computer Vision and Pattern Recognition. (2004)

10. Comaniciu, D., Meer, P.: Mean shift analysis and applications. In: Int. Conf. Computer Vision. (1999) 1197-1203

11. Lee, Y., Hara, T., Fujita, H., Itoh, S., Ishigaki, T.: Automated detection of pulmonary nodules in helical CT images based on an improved template-matching technique. IEEE Trans. Medical Imaging 20 (2001) 595-604

12. Comaniciu, D.: An algorithm for data-driven bandwidth selection. IEEE Trans. Pattern Anal. Machine Intell. 25 (2003) 281-288 